\title{
GENOME SEQUENCE OF BACILLUS VELEZENSIS W1, A STRAIN WITH STRONG ACARICIDAL ACTIVITY AGAINST TWO-SPOTTED SPIDER MITE (TETRANYCHUS URTICAE)
}

\author{
LI, X. Y. ${ }^{1,2}-$ MunIR, S. ${ }^{1}-$ CUI, W. Y. ${ }^{1}-$ He, P. J. ${ }^{1}-$ YANG, J. ${ }^{3}-$ He, P. F. ${ }^{1,2}-$ WU, Y. X. ${ }^{1,2}-$ \\ WANG, Y. H. ${ }^{3 *}-$ HE, Y. Q. ${ }^{12^{*}}$ \\ ${ }^{1}$ Yunnan Agricultural University, Kunming 650201, China \\ ${ }^{2}$ National and Local Joint Engineering Research Center for Screening and Application of \\ Microbial Strains, Kunming 650217, China
}

${ }^{3}$ Key Laboratory of Economic Plants and Biotechnology and Yunnan Key Laboratory for Wild Plant Resources, Chinese Academy of Sciences, Kunming 650201, China

*Corresponding authors

e-mail:wangyuehu@mail.kib.ac.cn (Y.H.Wang),ynfh2007@163.com (Y.Q.He)

(Received $26^{\text {th }}$ Nov 2018; accepted $28^{\text {th }}$ Jan 2019)

\begin{abstract}
Bacillus velezensis W1, isolated from two-spotted spider mites that had died naturally, is a patented strain with strong capability to cause mortality of the phytophagous mite Tetranychus urticae. The whole genome of W1 was completely sequenced with a combination of an Illumina Miseq platform (400-bp paired-end) with $2 \times 250$ bases and a Pacific Biosciences (PaBio) RS II Single Molecule Real Time (SMRT) sequencing platform using a 20 kb SMRTbell ${ }^{\mathrm{TM}}$ template library. Here, we report the complete genome sequence of B. velezensis $\mathrm{W} 1$, including one circular chromosome of $4,237,431$ bp encoding 4,352 genes with GC content of $45.84 \%$, providing insights into the genomic basis of its acaricidal activity and facilitating its application in red spider mite biocontrol.
\end{abstract}

Keywords: Bacillus, two-spotted spider mite, biocontrol, whole genome, Acaricides

\section{Introduction}

The two-spotted spider mite, Tetranychus urticae Koch, is one of the most polyphagous arthropod herbivores and feeds on more than 1,100 plant species belonging to more than 140 different plant families including species known to produce toxic compounds. It is a major pest in field crops, destroying both annual and perennial crops (Grbic et al., 2011). The use of chemical insecticides and acaricides are currently the primary control method of T. urticae (Jafari et al., 2016). The frequent application of these chemicals, paired with several biological aspects of this species, such as its short life cycle, high fecundity and arrhenotokous reproduction, has led to the mites developing resistance to the most pesticide groups (Minazzi et al., 2016). Additionally, chemical pesticides can have unintended consequences, impacting environmental quality, food safety, human health, and biodiversity (Yang et al., 2016).

Recently, biological control agents in the form of parasites, predators and pathogens have gained a lot of attention. Biological control of spider mites using predators or parasites is widely practiced, particularly the use of phytoseiid mites, which are currently sold worldwide (Funayama et al., 2015). Entomopathogenic fungi or entomogenous fungi such as Hirsutella thompsonii Fisher (El-Sharabasy, 2015), Neozygites floridana Weiser and Muma (Klingen et al., 2008), Beauveria bassiana 
(Balsamo) Vuillemin (Ullah and Lim, 2015), Verticillium lecanii (Zimm.) (Seiedy, 2015), and Isaria cateniannulata (Zhang et al., 2016) are currently the most studied biological control agents of spider mites. Few bacteria, however, have been reported as biological control agents of spider mites owing to the piercing-sucking mouthparts of the mites, which make it difficult for bacteria to infect them. In recent years, researchers have concentrated on intracellular organisms such as Wolbachia that may cause distorted sex ratio in the mite offspring, thereby impacting population (Chen et al., 2016), and toxin-producing bacteria such as Bacillus thuringiensis that can produce crystal proteins called $\delta$-endotoxins that are commonly used as a biological acaricides (Neethu et al., 2016). Additional research has been carried out on potential acaricide producing strains such as Pseudomonas putida (Aksoy et al., 2008).

Bacillus velezensis W1 (W1 hereafter), isolated from two-spotted spider mite that had died naturally, is a patented strain (Patent no; ZL201610096541.8) that has a strong capability to cause mortality of the phytophagous mite T. urticae (Li et al., 2018). This strain has the potential to be a safe and eco-friendly acaricide. To further explore its biocontrol ability and to reveal its acaricidal activity mechanism, we carried out the complete genome sequencing and analysis of W1.

\section{Materials and methods}

\section{Bacterial strain and culture condition}

B. velezensis $\mathrm{W} 1$ is a Gram-positive rod shaped bacterium averaging $2.5 \mu \mathrm{m}$ in length and $1 \mu \mathrm{m}$ in width (Fig. $1 a$ ). W1 spores are centrally located and average 1.3 $\mu \mathrm{m}$ in length (Fig. $1 b$ ). The bacteria could grow rapidly in Luria Bertani (LB) liquid medium reaching the stationary phase after $12 \mathrm{~h}$ at $35^{\circ} \mathrm{C}$. By comparison, the growth rate in LB solid medium was much slower with the stationary phase attained at $24 \mathrm{~h}$. Optimum growth occur at a temperature $35^{\circ} \mathrm{C}$ and $\mathrm{pH} 8.0$ (Table 1). The colony morphology of strain $\mathrm{W} 1$ grown in solid LB medium is circular convex with undulate beige-opaque margins (Fig. 1c).

\section{Phylogenetic analysis}

Phylogenetic tree of the B. velezensis W1 and other related taxa was constructed with MEGA 7.0 (Kumar et al., 2016) using Neighbor-Joining method (Saitou and Nei, 1987) based on gyrB gene, and the distances were computed using the Maximum Composite Likelihood method (Tamura et al., 2004), with 1000 replications in the bootstrap test. Bootstrap confidence levels $>30 \%$ are indicated at the internodes. The scale bar indicates nucleotide substitutions per nucleotide position.

\section{Genome sequencing information}

\section{Genome project history}

Strain W1 was selected for sequencing due to its ability to cause mortality of the phytophagous mite, Tetranychus urticae. The whole genome was deposited in GenBank under the accession number CP028375. Genome sequencing and assembly was performed at the Wuhan Genoseq Technology Co., Ltd, Wuhan, China. The summary of the project information is shown in Table 2. 


$$
\text { - } 2691 \text { - }
$$

Table 1. Classification and general features of Bacillus velezensis W1 (Field et al., 2008)

\begin{tabular}{|c|c|c|c|}
\hline MIGS ID & Property & Term & Evidence code $^{a}$ \\
\hline & Classification & Domain Bacteria & TAS (Woese et al., 1990) \\
\hline & & Phylum Firmicutes & TAS (Gibbonst and Murray, 1978) \\
\hline & & Class Baclli & TAS (Oren and Garrity, 2016) \\
\hline & & Order Bacillales & TAS (Skerman et al., 1980) \\
\hline & & Family Bacillaceae & TAS (Fischer, 1895) \\
\hline & & Genus Bacillus & TAS (Cohn, 1872) \\
\hline & & Species Bacillus velezensis & TAS (Dunlap et al., 2016) \\
\hline & & (Type) strain: $W 1$ (CP028375) & \\
\hline & Gram stain & Positive & IDA \\
\hline & Cell shape & Rod & IDA \\
\hline & Motility & Motile & IDA \\
\hline & Sporulation & not reported & \\
\hline & Temperature range & $20-40{ }^{\circ} \mathrm{C}$ & IDA \\
\hline & Optimum temperature & $35^{\circ} \mathrm{C}$ & IDA \\
\hline & $\mathrm{pH}$ range; Optimum & $5-9 ; 8$ & IDA \\
\hline & Carbon source & Heterotrophic & IDA \\
\hline MIGS-6 & Habitat & Plant, spider mite & IDA \\
\hline MIGS-6.3 & Salinity & $14 \%(\mathrm{w} / \mathrm{v}) \mathrm{NaCl}$ & IDA \\
\hline MIGS-22 & Oxygen requirement & Aerobic & IDA \\
\hline MIGS-15 & Biotic relationship & free-living & IDA \\
\hline MIGS-14 & Pathogenicity & Not reported & \\
\hline MIGS-4 & Geographic location & China & NAS \\
\hline MIGS-5 & Sample collection & 2015 & NAS \\
\hline MIGS-4.1 & Latitude & $25^{\circ} 01^{\prime} \mathrm{N}$ & NAS \\
\hline MIGS-4.2 & Longitude & $102^{\circ} 19^{\prime} \mathrm{E}$ & NAS \\
\hline MIGS-4.4 & Altitude & 1835 & NAS \\
\hline
\end{tabular}

${ }^{a}$ Evidence codes - MIGS: The minimum information about genome sequence; IDA: Inferred from Direct Assay; TAS: Traceable Author Statement (i.e., a direct report exists in the literature); NAS: Nontraceable Author Statement (i.e., not directly observed for the living, isolated sample, but based on a generally accepted property for the species, or anecdotal evidence). These evidence codes are from the Gene Ontology project (Ashburner et al., 2000)
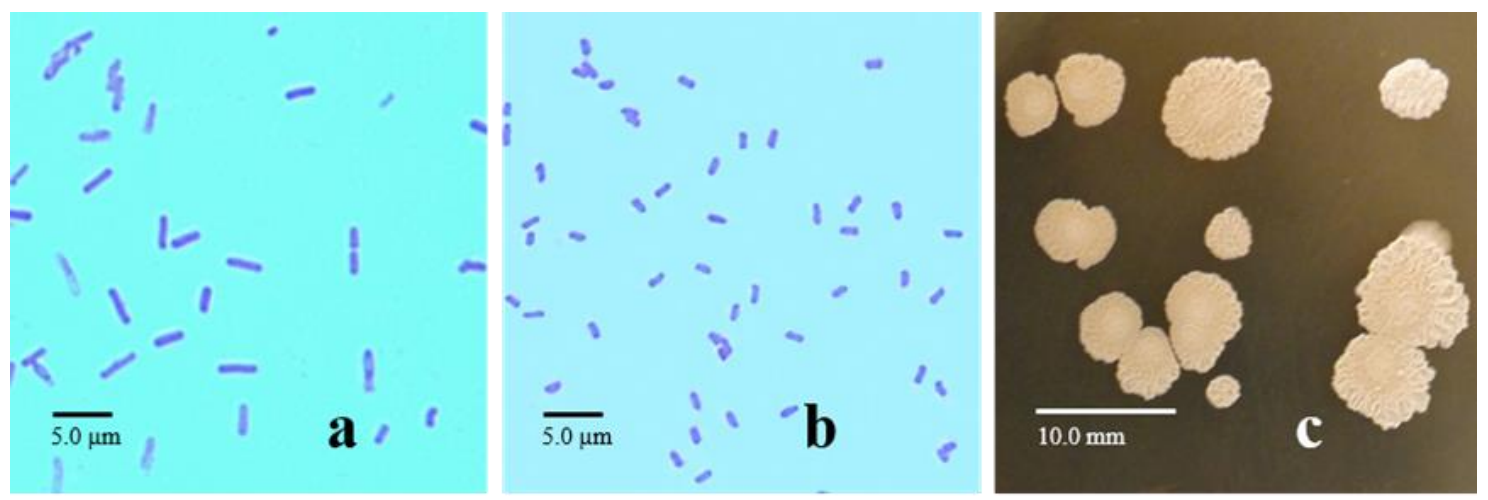

Figure 1. Cellular and colony morphology of Bacillus velezensis W1. a Vegetative W1 cells grown 72 h, Gram stained, and then at 100x magnification captured with light microscope. $\boldsymbol{b}$ W1 spores at 100x magnification captured light microscope. c Pure culture of W1 grown on nutrient agar plate 


$$
\text { - } 2692 \text { - }
$$

Table 2. Genome sequencing project information for Bacillus velezensis WI

\begin{tabular}{|c|c|c|}
\hline MIGS ID & Property & Term \\
\hline MIGS 31 & Finishing quality & Finished \\
\hline MIGS 28 & Libraries used & a $20 \mathrm{~kb}$ SMRTbell $^{\mathrm{TM}}$ template library \\
\hline MIGS 29 & Sequencing platforms & Illumina MiSeq + PacBio \\
\hline MIGS 31.2 & Fold coverage & $305 x$ \\
\hline MIGS 30 & Assemblers & A5-miseq version 20150522 \\
\hline \multirow[t]{6}{*}{ MIGS 32} & Gene calling method & NCBI Prokaryotic Genome Annotation Pipeline \\
\hline & Locus Tag & 1 \\
\hline & Genbank ID & СР028375 \\
\hline & Genbank Date of Release & June $28^{\text {th }}, 2018$ \\
\hline & GOLD ID & I \\
\hline & BIOPROJECT & PRJNA445958 \\
\hline \multirow[t]{2}{*}{ MIGS 13} & Source Material Identifier & Bacillus velezensis $\mathrm{W} 1$ \\
\hline & Project relevance & Biocontrol, Agriculture \\
\hline
\end{tabular}

\section{Growth conditions and genomic DNA preparation}

The genomic DNA of W1 was extracted using a QIAamp DNA mini kit (Qiagen, USA), according to the manufacturer's protocols.

\section{Genome sequencing and assembly}

The whole genome of W1 was completely sequenced with a combination of an Illumina Miseq platform (400-bp paired-end) with $2 \times 250$ bases and a Pacific Biosciences (PaBio) RS II Single Molecule Real Time (SMRT) sequencing platform using a $20 \mathrm{~kb}$ SMRTbell ${ }^{\mathrm{TM}}$ template library. Approximately $1,385.26 \mathrm{Mb}$ with 4,661,344 reads were generated from the Illumina Miseq and PacBio sequencings, respectively. The quality of these reads was assessed by the FastQC tool (http://www.bioinformatics.babraham.ac.uk/projects/fastqc) and low quality reads were filtered by Quake (Kelley et al., 2010) and AdapterRemoval (version 2.1.7) (Lindgreen, 2012). The clean reads were de novo assembled into a single contig with a genome coverage of 305 folds using A5-miseq version 20150522 (Coil et al., 2015), Canu v1.4 (Koren et al., 2017) and pilon v1.18 (Walker et al., 2014). Glimmer 3.02 (Delcher et al., 1999) was used to predict the open reading frames (ORFs).

\section{Genome annotation}

Software packages tRNAscan-SE v. 1.3.1 (Lowe and Eddy, 1997) and RNAmmer v. 1.2 (Lagesen et al., 2007) were used to predict tRNA and rRNA, respectively. The gene function annotations were based on BlastP similarity searches (E-Value $<10^{-6}$ ) against 5 databases: evolutionary genealogy of genes: Non-supervised Orthologous Groups (http://eggnogdb.embl.de/), Kncyclopedia of Genes and Genomes (http://www.genome.jp/kegg/), Non-Redundant GenBank Protein Database databases (www.ncbi.nlm.nih.gov/protein), Swiss-Prot (http://www.uniprot.org/), Gene Ontology Database (http://www.geneontology.org/). Circular genome map was created by cgview (Stothard and Wishart, 2005) with COG function annotation. 


$$
-2693-
$$

\section{Results and discussion}

\section{Sequence analysis using gyrB}

The phylogenetic analysis based on gyrB gene sequences using MEGA 7.0 [14] showed that $B$. velezensis $\mathrm{W} 1$ is evolutionarily positioned between $B$. velezensis and $B$. amyloliquefaciens (Fig. 2). In recent studies of genome sequencing and comparative genomics of B. velezensis NRRL B-41580, B. methylotrophicus KACC 13015 and $B$. amyloliquefaciens subsp. plantarum FZB42, it was established that these last two strains are heterotypic synonyms of B. velezensis (Dunlap et al., 2016), and based on our results, the classification of strain W1 was confirmed as a member of the species $B$. velezensis. The complete genome sequence of strain $B$. velezensis $\mathrm{W} 1$ is deposited at GenBank under accession number CP028375. The strain is available from China General Microbiological Culture Collection Center (CGMCC No. 11949).

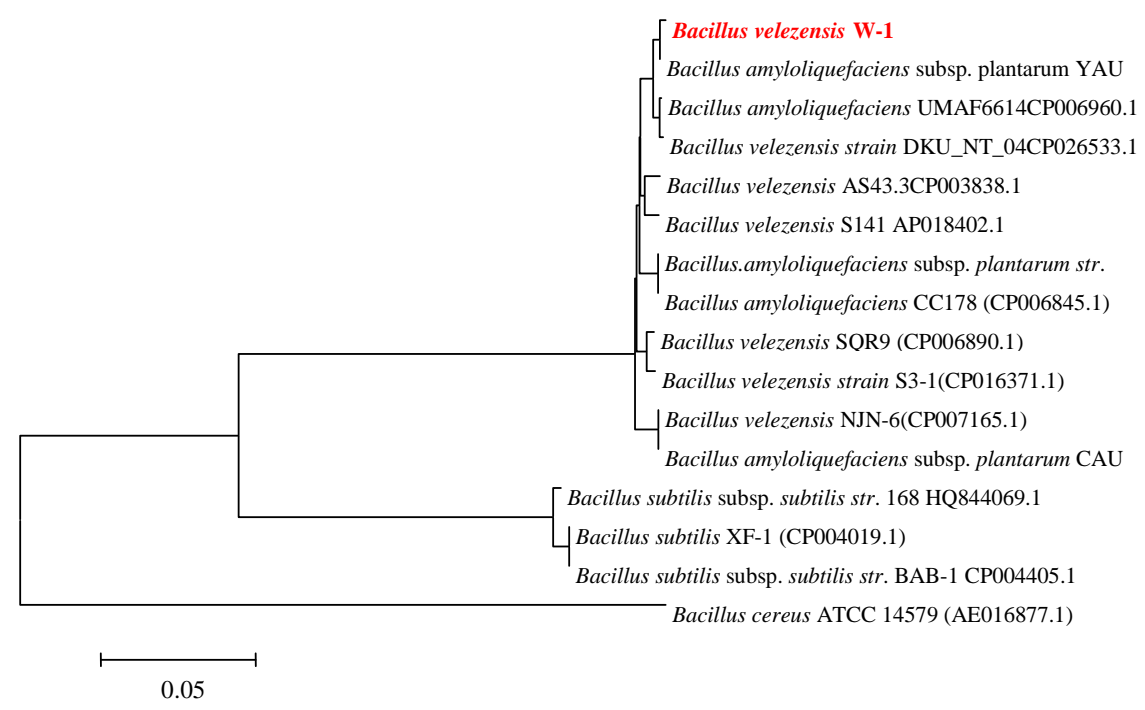

Figure 2. Phylogenetic trees of the B. velezensis WI and other related taxa

\section{Genome properties}

The complete genome of W1 consisted of one 4,237,431 bp circular chromosome with an average GC content of $45.84 \%$ without a plasmid. Totally, 4,404 protein-coding genes were predicted (Table 3; Fig. 3), along with 85 tRNA genes and 27 rRNA genes (Table 4; Fig. 3). Among these 4,404 protein-coding genes 4,352 ones (98.82\%) were annotated with predicted function (Table 5). There were $2879(65.37 \%)$ genes assigned to COG database (Table 6).

Table 3. Genome features of B. velezensis WI

\begin{tabular}{c|c}
\hline Features & Value \\
\hline Genome size (bp) & $4,237,431$ \\
Average G + C content (\%) & 45.84 \\
Protein-coding genes & 4,404 \\
Longest Protein-coding genes & 17,103 \\
Total size of Protein-coding genes & $3,761,991$ \\
Mean length of Protein-coding genes (bp) & 854 \\
Total size of Protein-coding genes \% of Genome (\%) & 88.78 \\
\hline
\end{tabular}




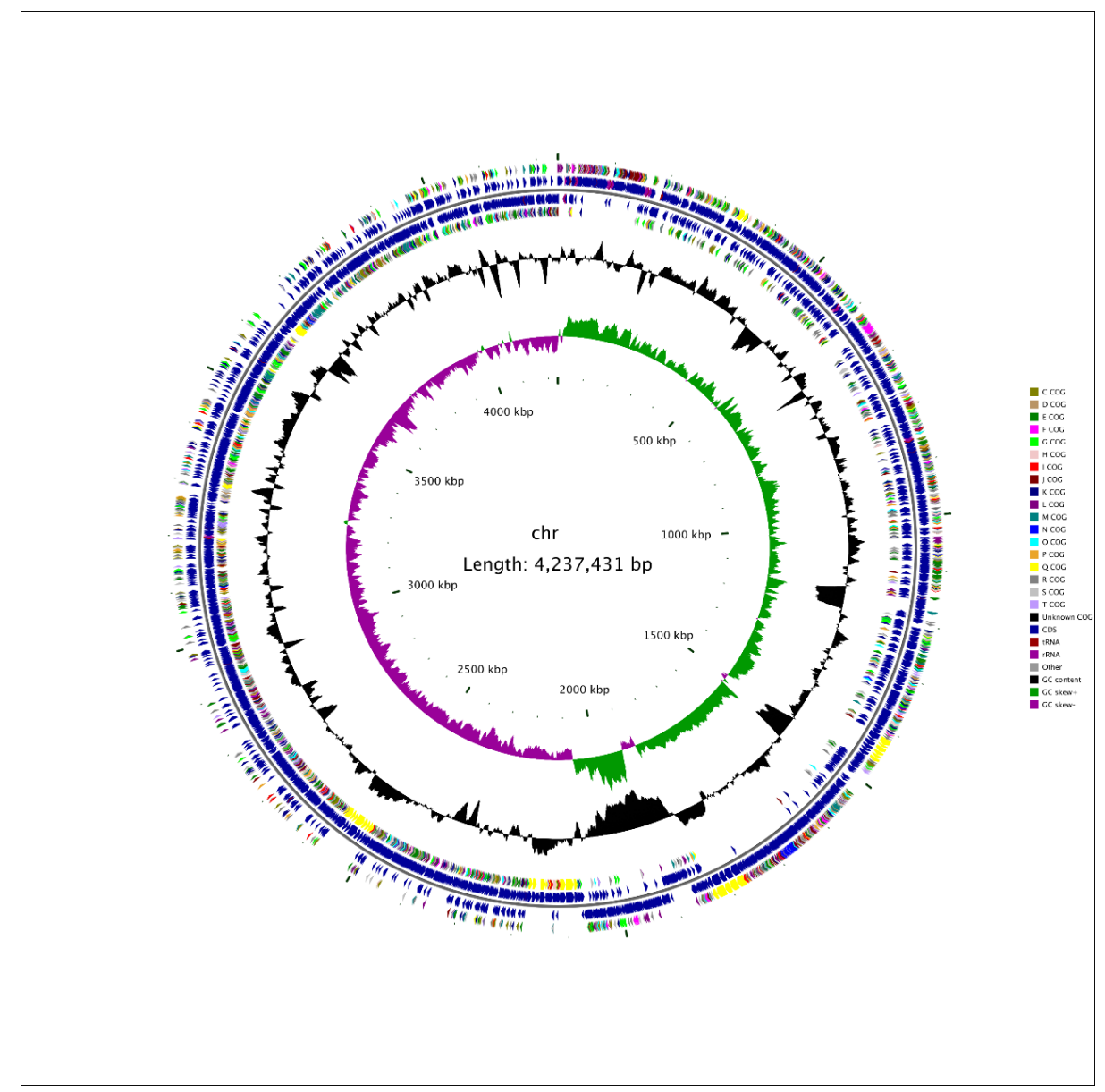

Figure 3. Circular genome graph of Bacillus velezensis W1. From inside to outside, the first circle represents the scale; the second circle represents the GC Skew; the third circle represents the GC content; the fourth and seventh circles represent each CDS's COG; the fifth and sixth circles represent the position of CDS, tRNA and rRNA on the genome

Table 4. Statistics of ncRNA prediction of protein-coding genes of B. velezensis WI

\begin{tabular}{c|c|c|c|c}
\hline ncRNA type & Copy & Average length (bp) & Total length (bp) & \% of genome \\
\hline 5s rRNA & 9 & 115 & 1,033 & 0.024 \\
16s rRNA & 9 & 1,570 & 14,128 & 0.333 \\
23s rRNA & 9 & 2,934 & 26,402 & 0.623 \\
tRNA & 85 & 77 & 6,568 & 0.155 \\
other ncRNA & 9 & 115 & 1,033 & 0.024 \\
\hline
\end{tabular}

Table 5. The statistics of gene function annotation of B. velezensis W1

\begin{tabular}{c|c|c}
\hline Database & Annotated number & \% of Genome \\
\hline NR & 4,352 & 98.82 \\
eggNOG & 2,879 & 65.37 \\
KEGG & 2,203 & 50.02 \\
Swiss-Prot & 3,785 & 85.94 \\
GO & 2,485 & 56.36 \\
\hline
\end{tabular}


Table 6. COG categories of B. velezensis WI

\begin{tabular}{c|c|c|c}
\hline Category & Description & Value & Percent (\%) \\
\hline B & Chromatin structure and dynamics & 1 & 0.02 \\
C & Energy production and conversion & 177 & 4.02 \\
D & Cell cycle control, cell division, chromosome partitioning & 33 & 0.75 \\
E & Amino acid transport and metabolism & 340 & 7.72 \\
F & Nucleotide transport and metabolism & 83 & 1.89 \\
G & Carbohydrate transport and metabolism & 250 & 5.68 \\
H & Coenzyme transport and metabolism & 125 & 2.84 \\
I & Lipid transport and metabolism & 119 & 2.70 \\
J & Translation, ribosomal structure and biogenesis & 144 & 3.27 \\
K & Transcription & 279 & 6.34 \\
L & Replication, recombination and repair & 133 & 3.02 \\
M & Cell wall/membrane/envelope biogenesis & 179 & 4.06 \\
N & Cell motility & 57 & 1.29 \\
O & Posttranslational modification, protein turnover, chaperones & 98 & 2.23 \\
P & Inorganic ion transport and metabolism & 205 & 4.66 \\
Q & Secondary metabolites biosynthesis, transport and catabolism & 116 & 2.63 \\
R & General function prediction only & 451 & 10.24 \\
S & Function unknown & 297 & 6.74 \\
T & Signal transduction mechanisms & 159 & 3.61 \\
U & Intracellular trafficking, secretion, and vesicular transport & 49 & 1.11 \\
V & Defense mechanisms & 63 & 1.43 \\
W & Extracellular structures & 3 & 0.07 \\
& Not in eggNoG & 34.63 \\
\hline
\end{tabular}

\section{Insights from the genome sequence}

The virulence factors of pathogenic bacteria (VFDB) of W1 were predicted using BLAST similarity searches (E-Value $<10-6)$ in Virulence Factors Database (http://www.mgc.ac.cn/VFs/) (Chen et al., 2012). W1 comprises several virulence factors (Table 7) which might all contribute to acaricidal activity, such as clpC, clpE, clpP, gale, lplA1, acpXL, bslA/yuaB, cps4I, and ureB.

There is a cluster of three collagen-related structural motif genes found in the genome, i.e., clpC, clpE and clpP. The genes of collagen-like proteins (clPs) have been identified in a broad range of bacteria, including some human pathogens and nonhuman pathogenic strains such as $B$. cereus and $B$. amyloliquefaciens which are reported to use as biocontrol agents (Zhao et al., 2015). The clPs are important for biofilm formation and bacterial adhesion to host cells and swimming motility (Zhao et al., 2016). In low-GC Gram-positive bacteria, the clpP protease is the main system involved in protein degradation (Vaz Cassenego et al., 2016), and play a role in both virulence and environmental adaptation, and acyldepsipeptides activated by ClpP core were effective in killing persister cells (Springer et al., 2016). 
Biofilms are surface-associated bacterial aggregates, in which bacteria are enveloped by polymeric substances known as the biofilm matrix. Biofilms of biocontrol strains were considered as some significant biocontrol properties, which provide the necessary proximity to the host so that the secretion of secondary metabolites featuring biocontrol activity from biofilm forming cells may act directly on the target (Kröber et al., 2016; Vlamakis et al., 2008). B. amyloliquefaciens is a Gram-positive bacterium that forms biofilms, which are created by a heterogeneous population of motile, matrix-producing and sporulating cells. One characteristic feature of biofilms is the extracellular matrix built from EPS combined with macromolecules like proteins and nucleic acids (Kröber et al., 2016). The bslA/yuaB was considered to stand for biofilm surface layer protein and be responsible for the hydrophobic layer on the surface of biofilms (Kobayashi and Iwano, 2012).

The chromosome encodes six chitin deacetylases (chr_orf00199, chr_orf01107, chr_orf03856, chr_orf05798, chr_orf01368, chr_orf02151), which are insect chitin degradation enzymes that catalyze the deacetylation of chitin to form chitosan, and enable the degradation of chitin in the midgut peritrophic membrane of many insects and have been identified as insect virulence factors (Yang et al., 2018; Yu et al., 2016).

Table 7. Predicted virulence factors of B. velezensis WI

\begin{tabular}{c|c|c|c}
\hline VFDB ID & Proteins & Annotation & Count \\
\hline VFG000079 (gi:16802278) & chr_orf00119 & clpC & 1 \\
VFG000080 (gi:16803037) & chr_orf01991 & clpE & 1 \\
VFG013286 (gi:16272302) & chr_orf05807 & galE & 1 \\
VFG002158 (gi:16802971) & chr_orf01464 & lplA1 & 1 \\
VFG011430 (gi:17987758) & chr_orf02383 & acpXL & 1 \\
VFG045350 (gi:16080160) & chr_orf04548 & bslA/yuaB & 1 \\
VFG000077 (gi:16804506) & chr_orf05117 & clpP & 1 \\
VFG001373 (gi:15900286) & chr_orf05282 & cps4I & 1 \\
VFG000270 (gi:15644702) & chr_orf05452 & ureB & 1 \\
\hline
\end{tabular}

\section{Conclusions}

This report described the complete genome sequence of B. velezensis W1. The species has biotechnological potential due to its capability to cause mortality of the phytophagous mite, Tetranychus urticae. Its acaricidal activity might be related to function of the virulence factors of pathogenic bacteria, like clpC, clpE, clpP, gale, lplA1, acpXL, bslA/yuaB, cps4I, and ureB, as well as collagen-related structural motif genes clpC, clpE and clpP. Biofilm and chitin deacetylases have been identified as biocontrol properties and insect virulence factors. Moreover, this bacterial strain can be used as microbial acaricides to control more and more acari in the field and greenhouse.

Acknowledgement. This work was supported by the National Natural Science Foundation of China (31660536).

Conflict of interests. The authors declare that they have no conflict of interests. 


\section{REFERENCES}

[1] Aksoy, H. M., Ozman-Sullivan, S. K., Ocal, H. et al. (2008): The effects of Pseudomonas putida biotype B on Tetranychus urticae (Acari: Tetranychidae). - Experimental and Applied Acarology 46: 223-30.

[2] Ashburner, M., Ball, C. A., Blake, J. A. et al. (2000): Gene ontology: tool for the unification of biology. - Nature Genetics 25: 25.

[3] Chen, L., Xiong, Z., Sun, L. et al. (2012): Toward the genetic diversity and molecular evolution of bacterial virulence factors. - Nucleic Acids Research 40: D641.

[4] Chen, Y. T., Zhang, Y. K., Du, W. X. et al. (2016): Geography has a greater effect than Wolbachia infection on population genetic structure in the spider mite, Tetranychus pueraricola. - Bulletin of Entomological Research 106: 685-94.

[5] Cohn, F. (1872): Untersuchungen über Bakterien. - Beitr Biol Pflanz 1: 127-224.

[6] Coil, D., Jospin, G., Darling, A. E. (2015): A5-miseq: an updated pipeline to assemble microbial genomes from Illumina MiSeq data. - Bioinformatics 31: 587-9.

[7] Delcher, A. L., Harmon, D., Kasif, S. et al. (1999): Improved microbial gene identification with GLIMMER. - Nucleic Acids Research 27: 4636-4641.

[8] Dunlap, C. A., Kim, S. J., Kwon, S. et al. (2016): Bacillus velezensis is not a later heterotypic synonym of Bacillus amyloliquefaciens; Bacillus methylotrophicus, Bacillus amyloliquefaciens subsp. plantarum and 'Bacillus oryzicola' are later heterotypic synonyms of Bacillus velezensis based on phylogenomics. - International Journal of Systematic and Evolutionary Microbiology 66: 1212-1217.

[9] El-Sharabasy, H. M. (2015): Laboratory evaluation of the effect of the entomopathogenic fungi, Hirsutella thompsonii and Paecilomyces fumosoroseus, against the citrus brown mite, Eutetranychus orientalis (Acari: Tetranychidae). - Plant Protection Science 51: 3945.

[10] Field, D., Garrity, G., Gray, T. et al. (2008): The minimum information about a genome sequence (MIGS) specification. - Nature Biotechnology. 26: 541.

[11] Fischer. (1895): Untersuchungen über Bakterien. - Jahrbücher für Wissenschaftliche Botanik 27: 1-163.

[12] Funayama, K., Komatus, M., Sonoda, S. et al. (2015): Management of apple orchards to conserve generalist phytoseiid mites suppresses two-spotted spider mite, Tetranychus urticae (Acari: Tetranychidae). - Experimental and Applied Acarology 65: 43-54.

[13] Gibbonst, N. E., Murray, R. G. E. (1978): Proposals concerning the higher taxa of bacteria. - International Journal of Systematic Bacteriology 28: 1-6.

[14] Grbic, M., Van, L. T., Clark, R. M. (2011): The genome of Tetranychus urticae reveals herbivorous pest adaptations. - Nature 479: 487-492.

[15] Jafari, S., Fathipour, Y., Faraji, F. et al. (2016): Demographic response to constant temperatures in Neoseiulus barkeri (Phytoseiidae) fed on Tetranychus urticae (Tetranychidae). - Systematic and Applied Acarology 15: 83-99.

[16] Kelley, D. R., Schatz, M. C., Salzberg, S. L. (2010): Quake: quality-aware detection and correction of sequencing errors. - Genome Biology 11: R116.

[17] Klingen, I., Waersted, G., Westrum, K. (2008): Overwintering and prevalence of Neozygites floridana (Zygomycetes: Neozygitaceae) in hibernating females of Tetranychus urticae (Acari: Tetranychidae) under cold climatic conditions in strawberries. - Experimental and Applied Acarology 46: 231-245.

[18] Kobayashi, K., Iwano, M. (2012): BslA(YuaB) forms a hydrophobic layer on the surface of Bacillus subtilis biofilms. - Molecular Microbiology 85: 51-66.

[19] Koren, S., Walenz, B. P., Berlin, K. et al. (2017): Canu: scalable and accurate long-read assembly via adaptive k-mer weighting and repeat separation. - Genome Research 27: 722. 
[20] Kröber, M., Verwaaijen, B., Wibberg, D. et al. (2016): Comparative transcriptome analysis of the biocontrol strain Bacillus amyloliquefaciens FZB42 as response to biofilm formation analyzed by RNA sequencing. - Journal of Biotechnology 231: 212-223.

[21] Kumar, S., Stecher, G., Tamura, K. (2016): MEGA7: Molecular Evolutionary Genetics Analysis Version 7.0 for bigger datasets. - Molecular Biology and Evolution 33: 1870.

[22] Lagesen, K., Hallin, P., Rødland, E. A. (2007): RNAmmer: consistent and rapid annotation of ribosomal RNA genes. - Nucleic Acids Research 35: 3100-8.

[23] Li, X. Y., Wang, Y. H., Yang, J. et al. (2018): Acaricidal activity of cyclodipeptides from Bacillus amyloliquefaciens W1 against Tetranychus urticae. - Journal of Agricultural and Food Chemistry 66(39): 10163-10168.

[24] Lindgreen, S. (2012): Adapter Removal: easy cleaning of next-generation sequencing reads. - BMC Research Notes 5: 337.

[25] Lowe, T. M., Eddy, S. R. (1997): tRNAscan-SE: a program for improved detection of transfer RNA genes in genomic sequence. - Nucleic Acids Research 25: 955-64.

[26] Minazzi, S. R. S., Sato, M. E., Santos, T. L. (2016): Stability and fitness costs associated with etoxazole resistance in Tetranychus urticae (Acari: Tetranychidae). - Experimental and Applied Acarology 69: 413-425.

[27] Neethu, K., Priji, P., Unni, K. (2016): New Bacillus thuringiensis strain isolated from the gut of Malabari goat is effective against Tetranychus macfarlanei. - Journal of Applied Entomology 140: 187-98.

[28] Oren, A., Garrity, G. M. (2016): List of new names and new combinations previously effectively, but not validly, published. - International Journal of Systematic and Evolutionary Microbiology 66: 4299-4305.

[29] Saitou, N., Nei, M. (1987): The neighbor-joining method: a new method for reconstructing phylogenetic trees. - Molecular Biology and Evolution 4: 406.

[30] Seiedy, M. (2015): Compatibility of Amblyseius swirskii (Acari: Phytoseiidae) and Beauveria bassiana for biological control of Trialeurodes vaporariorum (Hemiptera: Aleyrodidae). - Systematic and Applied Acarology 20: 731-8.

[31] Skerman, V. B. D., McGowan, V., Sneath, P. H. A. (1980): Approved lists of bacterial names. - International Journal of Systematic Bacteriology 30: 225-420.

[32] Springer, M. T., Singh, V. K., Cheung, A. L. (2016): Effect of clpP and clpC deletion on persister cell number in Staphylococcus aureus. - Journal of Medical Microbiology 65: 848-57.

[33] Stothard, P., Wishart, D. S. (2005): Circular genome visualization and exploration using CGView. - Bioinformatics 21(4): 537-539.

[34] Tamura, K., Nei, M., Kumar, S. (2004): Prospects for inferring very large phylogenies by using the neighbor-joining method. - Proceedings of the National Academy of Sciences 101: 11030-11035.

[35] Ullah, M. S., Lim, U. T. (2015): Laboratory bioassay of Beauveria bassiana against Tetranychus urticae (Acari: Tetranychidae) on leaf discs and potted bean plants. Experimental and Applied Acarology 65: 307-18.

[36] Vaz Cassenego, A. P., Moreira de Oliveira, N. E., Laport, M. S. (2016): The CtsR regulator controls the expression of clpC, clpE and clpP and is required for the virulence of Enterococcus faecalis in an invertebrate model. - Antonie Van Leeuwenhoek International Journal of General and Molecular Microbiology 109: 1253-1259.

[37] Vlamakis, H., Aguilar, C., Losick, R. et al. (2008): Control of cell fate by the formation of an architecturally complex bacterial community. - Genes and Development 22: 945953.

[38] Walker, B. J., Abeel, T., Shea, T. et al. (2014): An integrated tool for comprehensive microbial variant detection and genome assembly improvement. - Plos One 9: e112963.

[39] Woese, C. R., Kandler, O., Wheelis, M. L. (1990): Towards a natural system of organisms: proposal for the domains Archaea, Bacteria, and Eucarya. - Proceedings of the National Academy of Sciences 87: 4576-9. 
[40] Yang, L., Elbakidze, L., Marsh, T. et al. (2016): Primary and secondary pest management in agriculture: balancing pesticides and natural enemies in potato production. Agricultural Economics 47: 609-619.

[41] Yang, W. J., Xu, K. K., Yan, X. et al. (2018): Functional characterization of chitin deacetylase 1 gene disrupting larval\&ndash;pupal transition in the drugstore beetle using RNA interference. - Comparative Biochemistry and Physiology Part B: Biochemistry and Molecular Biology 219-220: 10-16.

[42] Yu, H. Z., Liu, M. H., Wang, X. Y. et al. (2016): Identification and expression profiles of chitin deacetylase genes in the rice leaf folder, Cnaphalocrocis medinalis. - Journal of Asia-Pacific Entomology 19: 691-6.

[43] Zhang, X., Jin, D., Zou, X. et al. (2016): Laboratory and field evaluation of an entomopathogenic fungus, Isaria cateniannulata strain 08XS-1, against Tetranychus urticae (Koch). - Pest Management Science 72: 1059-1066.

[44] Zhao, X., Wang, Y., Shang, Q. et al. (2015): Collagen-like proteins (ClpA, ClpB, ClpC, and $\mathrm{ClpD}$ ) are required for biofilm formation and adhesion to plant roots by Bacillus amyloliquefaciens FZB42. - Plos One 10(2): e0117414.

[45] Zhao, X., Wang, R., Shang, Q. et al. (2016): The new flagella-associated collagen-like proteins ClpB and ClpC of Bacillus amyloliquefaciens FZB42 are involved in bacterial motility. - Microbiological Research 184: 25-31. 